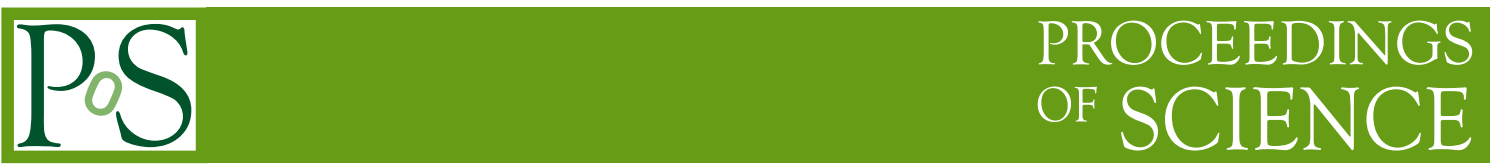

\title{
Measuring TeV neutrinos with FASER $v$ in LHC Run 3
}

\section{Tomoko Ariga ${ }^{a, *}$ and the FASER Collaboration}

${ }^{a}$ Kyushu University, Nishi-ku, 819-0395 Fukuoka, Japan

E-mail: tomoko.ariga@cern.ch

Neutrinos from a particle collider have never been directly detected. FASER $v$ at the Large Hadron Collider (LHC) is designed to detect such neutrinos for the first time and study their cross sections at $\mathrm{TeV}$ energies-at present, no such measurements are available at such high energies. In 2018, during LHC Run 2, we installed a pilot detector 480-m downstream of the ATLAS interaction point. In this pilot run, proton-proton collision data of $12.2 \mathrm{fb}^{-1}$ at a center-of-mass energy of $13 \mathrm{TeV}$ were collected. We observed the first candidate vertices, which were consistent with neutrino interactions. A $2.7 \sigma$ excess of neutrino-like signal above the background was measured. This milestone opens a new avenue for studying neutrinos at the existing and future high-energy colliders. During LHC Run 3, which will commence in 2022, we will deploy an emulsion detector with a target mass of 1.1 tons, coupled with the FASER magnetic spectrometer. This will yield $\sim 2,000 v_{e}, \sim 6,000 v_{\mu}$, and $\sim 40 v_{\tau}$ interactions in the detector. Herein, we present the status and plan of FASER $v$ and report neutrino detection in the 2018 data.

*** The European Physical Society Conference on High Energy Physics (EPS-HEP2021), ***

*** 26-30 July $2021 * * *$

*** Online conference, jointly organized by Universität Hamburg and the research center DESY ***

${ }^{*}$ Speaker 


\section{Physics motivations}

There has been a longstanding interest in detecting neutrinos produced at particle colliders, although collider neutrinos have not been directly detected. In Run 3 of the Large Hadron Collider (LHC), proton-proton collisions at a center-of-mass energy of $14 \mathrm{TeV}$ and with an expected integrated luminosity of $150 \mathrm{fb}^{-1}$ will produce a high-intensity beam of $O\left(10^{12}\right)$ neutrinos in the far-forward direction with mean interaction energy of $\sim 1 \mathrm{TeV}$. In the ForwArd Search ExpeRiment (FASER), FASER $v$ [1] is designed to detect these neutrinos and study their properties. The proposal for FASER $v$ [2] was approved in December 2019, and it is being installed 480-m downstream of the ATLAS interaction point. Beam exposure and data taking will be started from 2022. FASER $v$ is deployed on the beam collision axis to maximize the flux of all three neutrino $\left(v_{e}, v_{\mu}, v_{\tau}\right)$, thereby allowing FASER $v$ to measure their interaction cross sections in the TeV energy range, which is currently unexplored. Figure 1 shows the existing constraints on neutrino charged-current interaction cross sections and the expected energy spectra of the neutrinos interacting in FASER $v$. The FASER $v$ measurements will probe the gap between accelerator measurements $\left(E_{v}<360 \mathrm{GeV}\right)$ [3] and IceCube data $\left(E_{v}>6.3 \mathrm{TeV}\right)[4-6]$ for muon neutrinos and will extend the existing cross section measurements to considerably higher energies for electron and tau neutrinos. In addition to the measurements of charged-current interactions, neutral-current interactions can be measured. Such measurements may provide a new limit on nonstandard interactions of neutrinos to complement the existing limits known from other tests [7].

From another perspective, forward particle production, which is poorly constrained by the other LHC experiments, can be studied with FASER $v$. In particular, FASER $v$ measurements of high-energy electron neutrinos, which mainly originate from charm decays, can provide the first data on forward charm production. In the case of neutrino telescopes such as IceCube, accelerator measurements of high-energy and large-rapidity charm production are necessary for the precise measurement of the cosmic neutrino flux. As a 14-TeV proton-proton collision corresponds to a $100-\mathrm{PeV}$ proton interaction in the fixed-target mode, a direct measurement using FASER $v$ can provide important basic data for current and future high-energy neutrino telescopes.
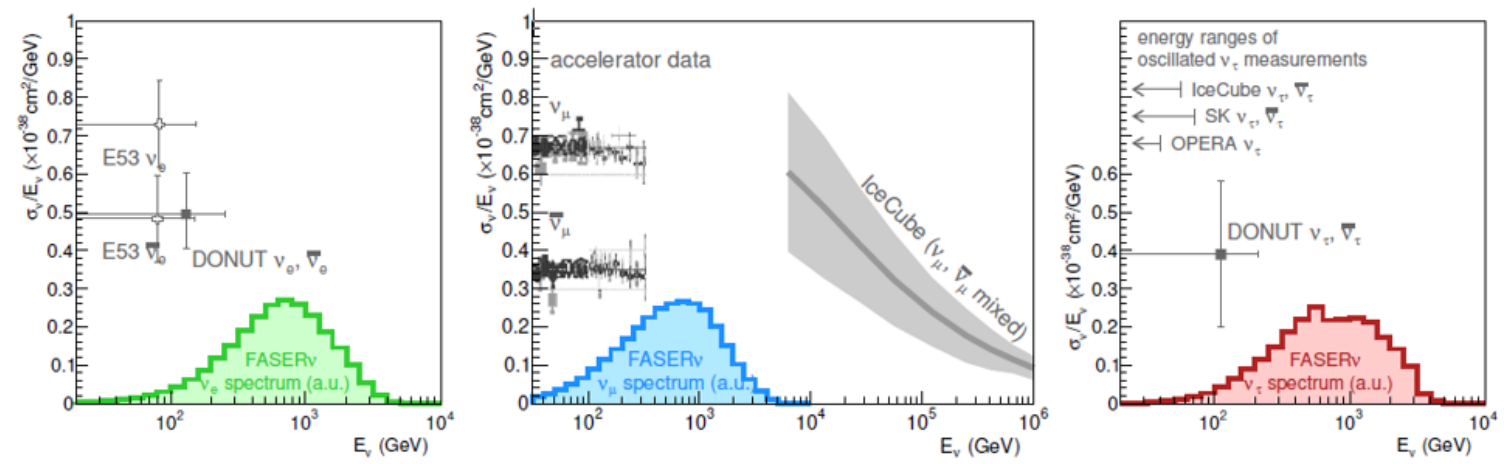

Figure 1: Existing constraints on neutrino charged-current interaction cross sections, and the expected energy spectra of neutrinos interacting in the FASER $v$ detector [1]. 


\section{Detector and neutrino beam}

The FASER $v$ detector is located in front of the main FASER detector [8] along the beam collision axis to maximize the interaction rate of neutrinos of all three flavors. As shown in Figure 2, the detector has a veto station, an emulsion/tungsten detector, and an interface tracker (IFT) connected to the FASER spectrometer. The emulsion/tungsten detector is designed to identify different lepton flavors. It has sufficient target material to identify muons, finely sampled detection layers to identify electrons and to distinguish them from gamma rays, and good position and angular resolutions to detect tau and charm decays. Additionally, the detector can measure muon and hadron momenta and energy of an electromagnetic shower and provide an estimate neutrino energy. The IFT is located downstream of the emulsion/tungsten detector to make the global analysis, using both the emulsion/tungsten detector and the FASER spectrometer, and muon charge measurement possible.

The emulsion/tungsten detector is composed of a repeated structure of emulsion films interleaved with 1-mm-thick tungsten plates. The emulsion film has two emulsion layers, each $65-\mu \mathrm{m}$ thick. These layers are added onto both sides of a $210-\mu \mathrm{m}$-thick plastic base. The emulsion detector contains a total of 770 emulsion films, each of dimensions $25 \mathrm{~cm} \times 30 \mathrm{~cm}$, and a total tungsten mass of 1.1 tons.

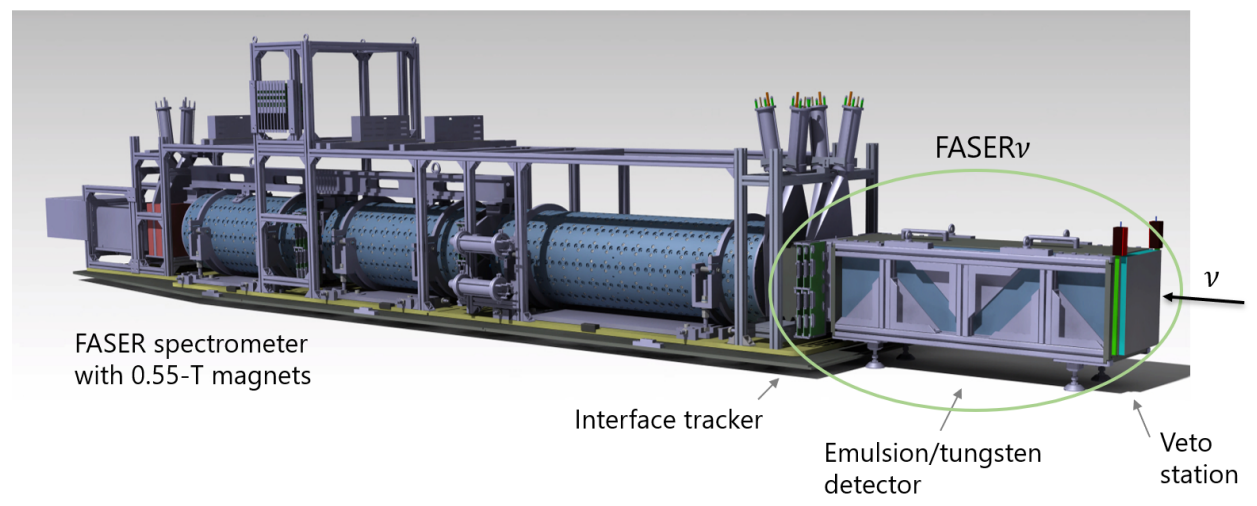

Figure 2: Sketch of the FASER detector, highlighting the FASER $v$ detector.

In March 2021, the main FASER detector was successfully installed into TI12 tunnel. Figure 3 shows a picture of the installed detector. FASER $v$ veto station and IFT were assembled, used for the test beam at $\mathrm{H} 2$ beamline in the CERN SPS North Area, and then installed in TI12 in November 2021. The emulsion/tungsten detector will be installed just before the exposure and will be replaced during planned technical stops. The production of emulsion gel and films is scheduled few months before each replacement.

The neutrinos at the LHC originate from the decays of forward-going hadrons, in particular, pions, kaons, hyperons, and charmed hadrons. A high-intensity beam of neutrinos is then produced in the far-forward direction. The expected numbers of neutrino interactions in FASER $v$ during LHC Run 3 are $\sim 2,000 v_{e}, \sim 6,000 v_{\mu}$, and $\sim 40 v_{\tau}$. The differences between the generators are verified and summarized in [9]. 


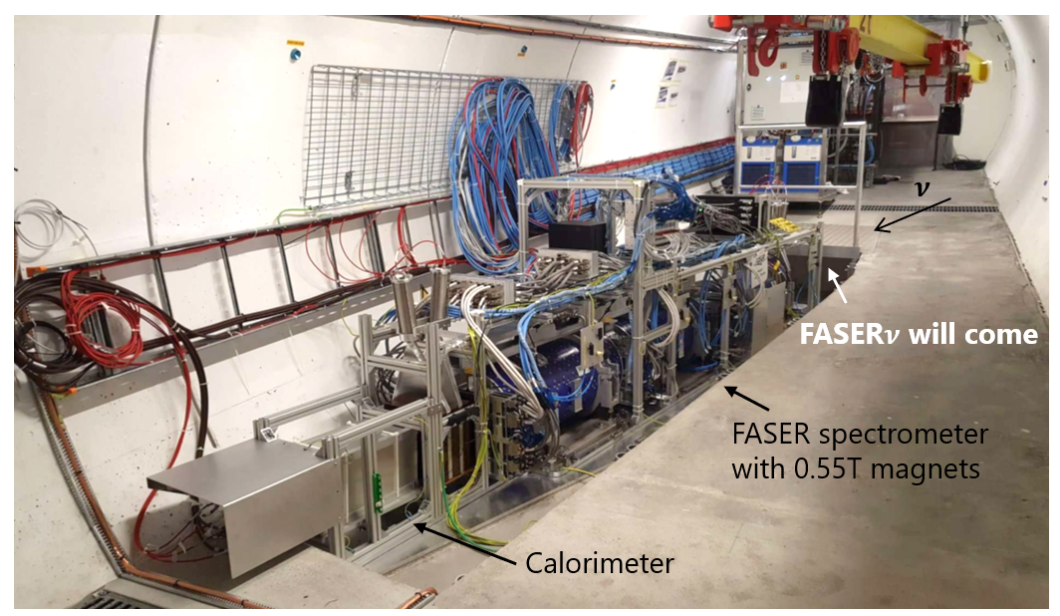

Figure 3: Picture of the main FASER detector installed in TI12 tunnel in March 2021.

\section{First neutrino interaction candidates in the 2018 data}

In 2018, a pilot run was performed in the LHC tunnel to demonstrate neutrino detection at the LHC for the first time. The pilot detector could not identify muons because its depth was only $0.6 \lambda_{\text {int }}$, which is much shorter than the $8 \lambda_{\text {int }}$ of the full FASER $v$ detector. The data from the pilot detector are used to prove the feasibility of neutrino measurements in this experimental environment.

We searched for neutrino interactions by analyzing the data corresponding to $11 \mathrm{~kg}$ of the target mass. We observed the first candidate vertices [10] to be consistent with neutrino interactions at the LHC. Figure 4 shows two candidate events. A $2.7 \sigma$ excess of neutrino-like signals over muon-induced backgrounds was measured. These results demonstrate the ability of FASER $v$ to detect neutrinos at the LHC and thus pave the way for future collider neutrino experiments.
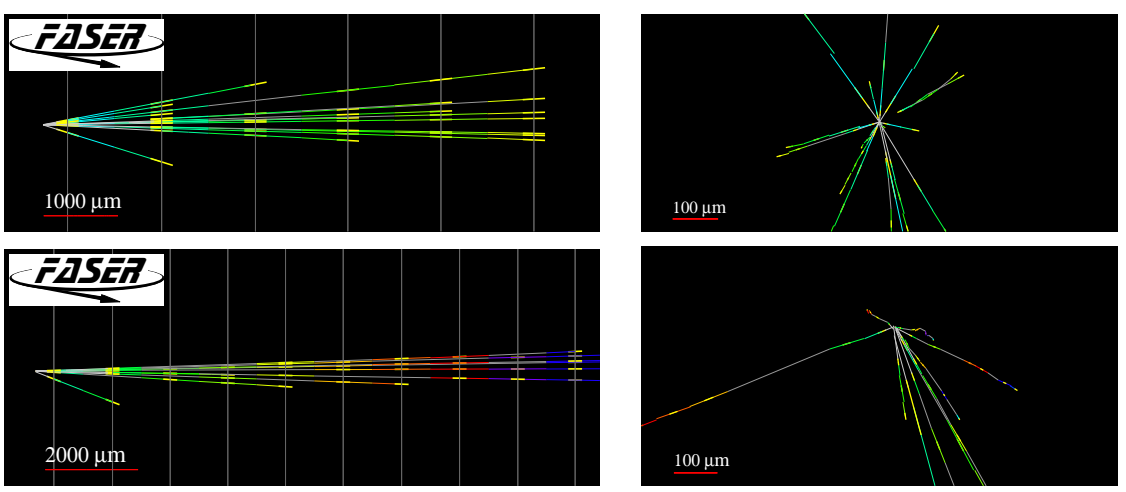

Figure 4: Event displays of two neutrino interaction candidates [10] in the $y-z$ projection longitudinal to the beam direction (left) and transverse to the beam direction (right). 


\section{Summary and prospects}

FASER $v$ is designed to detect collider neutrinos of all three flavors and provide new measurements of their cross sections at energies higher than those detected from any previous artificial sources. In the data taken in 2018 during LHC Run 2, we observed the first neutrino interaction candidates at the LHC. Currently, our preparations are ongoing for obtaining data in LHC Run 3. With a deeper detector and lepton identification capability, FASER $v$ will perform better than the pilot detector in 2018. In 2022-2024, during LHC Run 3, we expect to collect 10,000 flavor-tagged charged-current neutrino interactions, along with neutral-current interactions.

Toward the High-Luminosity LHC era, we are planning to establish FASER $v 2$ at a proposed facility, namely, the Forward Physics Facility [11], which is proposed to house a suite of experiments and to greatly enhance the physics potential of the LHC for searches related to physics beyond the standard model, neutrino physics, and QCD. FASER $v 2$ is designed to perform precision measurements of high-energy neutrinos, especially, for tau neutrinos, and heavy-flavor physics studies. The FASER $v 2$ detector is a much larger successor to FASER $v$. FASER $v 2$ is currently envisioned to be an emulsion-based detector with the target mass of 20 tons and to include a veto detector and interface detectors for the FASER2 spectrometer. The high muon background in the LHC tunnel may be the experimental limitation for FASER $v 2$. Hence, the possibility of sweeping away such muons using a magnetic field placed upstream of the detector is currently being explored.

\section{Acknowledgments}

The collaboration acknowledges CERN for the excellent performance of the LHC and the technical and administrative staff members at all FASER institutions. We gratefully acknowledge invaluable assistance from many groups at CERN, particularly, the Physics Beyond Colliders study group, the ATLAS Collaboration for providing the luminosity value, and the NA65/DsTau Collaboration for providing spare emulsion films for the 2018 run. This work was supported partly by Heising-Simons Foundation Grant Nos. 2018-1135, 2019-1179, and 2020-1840, Simons Foundation Grant No. 623683. This work was supported by JSPS KAKENHI Grant Nos. JP19H01909, JP20H01919, JP20K04004, JP20K23373, a research grant from the Mitsubishi Foundation, and the joint research program of the Institute of Materials and Systems for Sustainability.

\section{References}

[1] H. Abreu et al. (FASER Collaboration), Detecting and Studying High-Energy Collider Neutrinos with FASER at the LHC, Eur. Phys. J. C 80, 61 (2020), https://link. springer. com/article/10.1140/epjc/s10052-020-7631-5.

[2] H. Abreu et al. (FASER Collaboration), Technical Proposal: FASERv, CERN-LHCC-2019017, https://arxiv.org/abs/2001.03073.

[3] P. A. Zyla et al. (Particle Data Group Collaboration), Review of Particle Physics, PTEP 2020, 083C01 (2020), https://academic.oup.com/ptep/article/2020/8/083C01/ 5891211. 
[4] M. Aartsen et al. (IceCube Collaboration), Measurement of the multi-TeV neutrino cross section with IceCube using Earth absorption, Nature 551, 596 (2017), https: / /www . nature.com/ articles/nature24459.

[5] M. Bustamante and A. Connolly, Extracting the Energy-Dependent Neutrino-Nucleon Cross Section above $10 \mathrm{TeV}$ Using IceCube Showers, Phys. Rev. Lett. 122, 041101 (2019), https: //journals.aps.org/prl/abstract/10.1103/PhysRevLett.122.041101.

[6] R. Abbasi et al. (IceCube Collaboration), Measurement of the high-energy all-flavor neutrinonucleon cross section with IceCube, Phys. Rev. D 104, 022001 (2021), https: // journals . aps.org/prd/abstract/10.1103/PhysRevD. 104.022001.

[7] A. Ismail, R.M. Abraham, F. Kling, Neutral current neutrino interactions at FASERv, Phys. Rev. D 103, 056014 (2021), https://journals.aps.org/prd/abstract/10.1103/ PhysRevD. 103.056014.

[8] A. Ariga et al. (FASER Collaboration), Technical Proposal for FASER: ForwArd SearchExpeRiment at the LHC, CERN-LHCC-2018-036, https: //arxiv .org/abs/1812.09139.

[9] F. Kling and L. J. Nevay, Forward neutrino fluxes at the LHC, Phys. Rev. D 104, 113008 (2021), https://journals.aps.org/prd/abstract/10.1103/PhysRevD. 104.113008.

[10] H. Abreu et al. (FASER Collaboration), First neutrino interaction candidates at the LHC, Phy. Rev. D 104, L091101, https: //journals . aps . org/prd/abstract/10 .1103/ PhysRevD . 104.L091101.

[11] L.A. Anchordoqui et al., The Forward Physics Facility: Sites, Experiments, and Physics Potential, https://arxiv.org/abs/2109.10905. 